\title{
Proportional Differentiated Admission Control
}

\author{
Ronaldo M. Salles, Student Member, IEEE, and Javier A. Barria, Member, IEEE
}

\begin{abstract}
This letter presents a new admission control policy inspired in the framework of proportional differentiated services (PDS). While most of previous PDS has focused on average queueing delays and packet drops to differentiate the performance of adaptive applications, the proportional differentiation admission control (PDAC) differentiates inelastic traffic in terms of blocking probabilities. The PDAC is built up using asymptotic approximation theory, employs a class based approach, and conforms with the PDS requirements of predictability and controllability. Numerical experiments confirm a good performance of the approach.
\end{abstract}

Index Terms-Admission control, asymptotic approximation, proportional differentiated services.

\section{INTRODUCTION}

A DMISSION control is fundamental for the adequate support of inelastic traffic in data networks. This type of traffic is characterized by stringent quality of service (QoS) requirements and usually do not adapt to network congestion signals (e.g., real-time traffic). Regarding IP networks, the IETF IntServ and DiffServ architectures have emerged as the most prominent proposals to attend the QoS requirements posed by current network applications. However, while the first one may suffer from scalability problems, the latter may only provide qualitative assurances and lacks control to the network operator. Recently, a scalable, predictable (differentiation is independent of class load variations), and controllable (network operator should be able to adjust the relative QoS between classes) service architecture, the proportional differentiated services (PDS) [1], has been proposed as an alternative to overcome those problems.

Most PDS implementations do not employ admission control or resource reservation and so they do not serve for inelastic traffic. However a QoS network offering PDS may be also required to transmit inelastic traffic. The proportional differentiated admission control (PDAC) bridges that gap and brings the PDS framework to the context of inelastic traffic. It provides a predictable and controllable network service for inelastic traffic in terms of blocking probability, and uses a PDS equality to define such service.

\footnotetext{
Manuscript received September 18, 2003. The associate editor coordinating the review of this letter and approving it for publication was Prof. M. Devetsikiotis. The work of R. M. Salles was supported by the $C N P q$, Brazilian Government, under Grant 200049/99-2.

The authors are with the Department of Electrical and Electronic Engineering, Imperial College London, London SW7 2BT, U.K. (e-mail: r.salles@imperial.ac.uk; j.barria@imperial.ac.uk).

Digital Object Identifier 10.1109/LCOMM.2004.827384
}

\section{The PROPORTIONAL DifFENTIATION AdMISSION CONTROL (PDAC)}

\section{A. Environment and Formulation}

We consider a heterogeneous system where each connection has an associated bandwidth requirement $b$ that should be guaranteed end-to-end throughout its lifetime. To facilitate scalability, connections are grouped into classes that differ according to $b$. A connection $j$ should use class $k$ if $b_{k} \geq b_{j}$, and $b_{k}$ is the nearest to $b_{j}$ among all classes. Same class connections are aggregated into path flows so that they can be managed as a single entity inside the network The aggregate requirement of class $k$ flow is then $x_{k} b_{k}$, where $x_{k}$ is the number of class $k$ connections. A simple admission test $\left(x_{k}+1\right) b_{k} \leq C_{k}$ can be used to control the access to class partition $C_{k}$. This class-based approach is conservative since we assumed traffic to be inelastic and $b$ provided in an all-or-nothing basis. Signalling is mandatory, however, as long as we are conservative (i.e., use $b_{k}$ as the peak rate) per-flow management is reduced. There will be no need to allocate resources per-flow since class partition is likely to be over provisioned. Moreover, there will be no wasted resources given that all available resources can be used by other underlying PDS or best-effort traffic.

The above characterization is fundamentally different from the adaptive traffic commonly assumed in PDS. In our case, the only way to regulate the aggregated class flow and still meet bandwidth requirements is through blocking. Note also that blocking is a QoS parameter relevant for inelastic traffic. Each class $k$ incurs a certain blocking probability $\left(B_{k}\right)$ and according to proportional differentiation goal, PDAC is defined as

$$
\delta_{1} \cdot B_{1}=\delta_{2} \cdot B_{2}=\cdots=\delta_{K} \cdot B_{K}
$$

where, $\delta_{k}$ is the weight of class $k$. The weights work as priorities assigned to the classes, the larger the weight the higher the admittance priority. The strategy ensures a flexible traffic control for the network operator (controllability). The problem is then to find a resource allocation mechanism for the link capacity which guarantees (1).

The system can be modeled as a stochastic knapsack [2] of capacity $C$, which under a complete partitioning scheme (CP) is reduced to $K$ independent Erlang subsystems (exponential arrivals and general session holding times). Each class $k$ has an allotted partition $C_{k}$ and offered load $\rho_{k}$. The PDAC problem under $\mathrm{CP}$ is then

$$
\begin{aligned}
& \text { find: }\left(C_{1}, C_{2}, \ldots, C_{K}\right) \quad n_{k}=\left\lfloor C_{k} / b_{k}\right\rfloor \\
& \text { s.t.: } \delta_{1} \frac{\frac{\rho_{1}^{n_{1}}}{n_{1} !}}{\sum_{i=0}^{n_{1}} \frac{\rho_{1}^{i}}{i !}}=\delta_{2} \frac{\frac{\rho_{2}^{n_{2}}}{n_{2} !}}{\sum_{i=0}^{n_{2}} \frac{\rho_{2}^{i}}{i !}}=\cdots=\delta_{K} \frac{\frac{\rho_{K}^{n_{K}}}{n_{K} !}}{\sum_{i=0}^{n_{K}} \frac{\rho_{K}^{i}}{i !}} \\
& \quad \sum_{k=1}^{K} C_{k}=C
\end{aligned}
$$


where $\delta=\left(\delta_{1}, \delta_{2}, \ldots, \delta_{K}\right), \rho=\left(\rho_{1}, \rho_{2}, \ldots, \rho_{K}\right)$, and $b=$ $\left(b_{1}, b_{2}, \ldots, b_{K}\right)$ are given. The above problem is a huge combinatorial problem even for small instances. Moreover, it is not likely to have a feasible solution given the hard equality constraints on (3). Therefore we work on two different directions: $i$ ) find ways to reduce the complexity of the problem and $i i$ ) relax PDAC definition in order to avoid sharp equality constraints.

\section{B. Asymptotic Approximation}

From the early studies on the Erlang $B$ formulae [3], $B(n, \rho)$, it can be shown that blocking probability in the limiting regime (when $n \rightarrow \infty$ and $n / \rho<1$ ) takes the following form:

$$
\lim _{n \rightarrow \infty} B(n, \rho)=1-\frac{n}{\rho}
$$

where $n=C / b$. This result simply states that expressions can be strongly simplified when the capacity of links and offered loads are increased together: $n_{k} \rightarrow \infty\left(C_{k} \rightarrow \infty\right)$ and $\rho_{k}>n_{k}$. The condition can be satisfied in links used by a large number of flows, where each class partition $C_{k}$ is kept below $b_{k} \rho_{k}$.

Using asymptotic approximation, (3) becomes

$$
\begin{aligned}
\delta_{1}\left(1-\frac{C_{1}}{b_{1} \rho_{1}}\right) & =\delta_{2}\left(1-\frac{C_{2}}{b_{2} \rho_{2}}\right)=\cdots \\
& =\delta_{K}\left(1-\frac{C_{K}}{b_{K} \rho_{K}}\right)
\end{aligned}
$$

Taking the class of minimum weight $\left(\delta_{j} \leq \delta_{k}\right)$ as reference, the partitions that satisfy PDAC goal can be now obtained from,

$$
\begin{aligned}
C_{k} & =b_{k} \rho_{k}\left[1-\frac{\delta_{j}}{b_{j} \rho_{j} \delta_{k}}\left(b_{j} \rho_{j}-C_{j}\right)\right], \quad k \neq j \\
\sum_{k=1}^{K} C_{k} & \leq C
\end{aligned}
$$

Although the approximation allowed a significant reduction in the complexity of the PDAC problem, the feasibility issue still remains. For instance, to guarantee $C_{j}>0 \Rightarrow$

$$
\begin{aligned}
C_{k} & >b_{k} \rho_{k}\left(1-\frac{\delta_{j}}{\delta_{k}}\right) \\
& \Rightarrow C>\sum_{k=1}^{K} b_{k} \rho_{k}\left(1-\frac{\delta_{j}}{\delta_{k}}\right) .
\end{aligned}
$$

Therefore, given the capacity of the $\operatorname{link} C$, it is not always possible to solve the PDAC for general values of $b_{k}, \rho_{k}$, and $\delta_{k}$ even when considering the asymptotic approximation. The sharp equality constraints in the PDAC definition need to be relaxed.

\section{Problem Relaxation}

In addition to the asymptotic approximation, we relax the PDAC definition in order to avoid the equality in (1) and overcome the feasibility difficulty. To achieve that the min max operator is employed redefining the service goal, or in other words defining the RPDAC (Relaxed PDAC) problem

$$
\begin{aligned}
& \text { find: }\left(C_{1}, C_{2}, \ldots, C_{K}\right) \quad n_{k}=\left\lfloor C_{k} / b_{k}\right\rfloor \\
& \text { s.t.: } \min \max _{k}\left\{\delta_{k} B_{k}\right\} \quad k=1,2, \ldots, K
\end{aligned}
$$

$$
\sum_{k=1}^{K} C_{k} \leq C
$$

The rationale behind the min max operator is that while it tries to minimize the differences between $\delta_{k} B_{k}$ of the classes, it does not impose strict equality.

With the introduction of an extra variable $(\xi)$, the min max in (11) can also be written as:

$$
\xi^{*}=\min \left\{\xi \mid \delta_{k} B_{k} \leq \xi, k=1,2, \ldots, K\right\}
$$

Since there is no restriction on $\xi$ apart from being a real number, it is always possible to return a solution for (13). The blocking probability for class- $k$ traffic is given by $B_{k} \leq \xi^{*} / \delta_{k}$, such bound should be tolerated by the traffic. Note that PDAC offers absolute guarantees on bandwidth but not on blocking where the goal is to satisfy (1). Again applying the asymptotic approximation to $B_{k}$ in (13) we end up with the following low cost linear programming problem $(K+1$ variables and $2 K+1$ constraints),

$$
\min \left\{\xi \mid \delta_{k} C_{k}+\xi a_{k} \geq \delta_{k} a_{k}, C_{k}<a_{k}, \sum_{k=1}^{K} C_{k} \leq C\right\}
$$

where: $k=\{1, \ldots, K\}, a_{k}=b_{k} \rho_{k}$, and $\xi \in \mathbb{R}$. The class partitions $C_{k}$ returned from the solution of (14) provide an approximated implementation for the PDAC service.

\section{LOAD ESTIMATION}

In order to return the link partitions from the relaxed problem in (14) it is necessary to determine all class loads $\rho_{k}$ in advance. However, in a real scenario class loads are likely to change due to the dynamics involving session arrivals and departures. We propose a maximum likelihood estimator (MLE) for each $\rho_{k}$ that continuously estimate loads from network observed states $x_{k}(i)$ (number of class $k$ sessions in the system at time $t_{i}$ ). The Erlang formulae is used as the likelihood function,

$$
\begin{aligned}
p\left(x_{k}(i) ; \theta\right) & =\frac{\theta^{x_{k}(i)}}{x_{k}(i) !} G^{-1}(\theta) ; \\
G(\theta) & =\sum_{j=0}^{C} \frac{\theta^{j}}{j !}
\end{aligned}
$$

Taking past $N$ independent samples, we have

$$
p\left(x_{\boldsymbol{k}} ; \theta\right)=\theta^{\left[\sum_{i=0}^{N-1} x_{k}(i)\right]} G^{-N}(\theta) / \prod_{i=0}^{N-1} x_{k}(i) !
$$

The MLE is computed from

$$
\frac{\partial}{\partial \theta} \log p\left(\boldsymbol{x}_{\boldsymbol{k}} ; \theta\right)=\frac{1}{\theta} \sum_{i=0}^{N-1} x_{k}(i)-N \frac{\sum_{j=1}^{C} \frac{\theta^{j-1}}{(j-1) !}}{\sum_{j=0}^{C} \frac{\theta^{j}}{j !}}=0
$$

The fraction in the right term is simply the acceptance probability, thus the MLE is reduced to

$$
\theta=\sum_{i=0}^{N-1} x_{k}(i) / N\left(1-\hat{B}_{k}\right)
$$

The $N$ samples are collected during each interval $T$, and $\hat{B}_{k}$ directly computed through the ratio between rejected and total number of connection arrivals. A new PDAC problem is solved at each $T$ and new class partitions obtained. Using a receiver- 


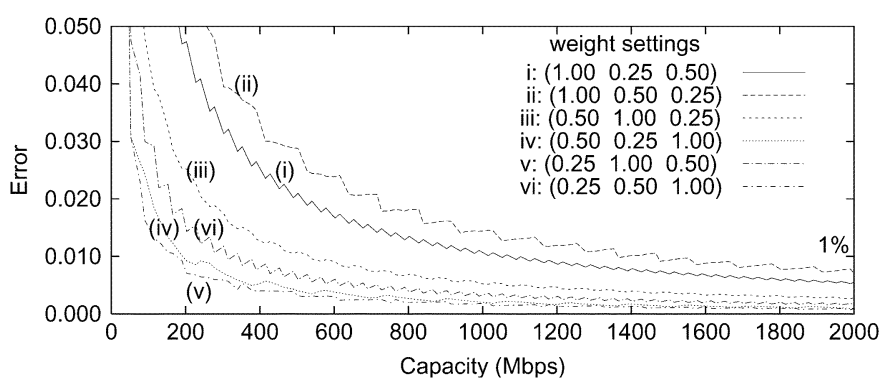

Fig. 1. PDAC errors for Exp1, system scaled from $\gamma=1$ to $\gamma=10^{3}$.

oriented signalling protocol (e.g., RSVP) the response for a request is acknowledged by the receiver and follows the backward path until it reaches the origin node. In this way, the loads can be computed from (18) by all links along the path, and the PDAC can be independently implemented on each link using only locally available information.

\section{NUMERICAL RESULTS}

The error incurred in any PDAC implementation can be calculated taking the class of unitary weight, say class-1, as reference $\left(\delta_{1}=1\right)$ and $\hat{\delta}_{k}=B_{1} / B_{k}$ as the blocking ratio.

$$
\text { error }=\sqrt{\frac{1}{K-1} \sum_{k=2}^{K}\left(\frac{\hat{\delta}_{k}-\delta_{k}}{\max \left\{\hat{\delta}_{k}, \delta_{k}\right\}}\right)^{2}} .
$$

Equation (19) quantifies the deviations a given implementation may produce from the goals set for PDAC (1), where error $=0$ implies in a precise implementation.

We carried out two experiments to evaluate the performance of a PDAC implementation based on asymptotic approximation. We used the same number of classes as observed in other PDS [1], in the first experiment (Exp1) we considered three classes of traffic while in the second (Exp2) we used four classes as shown in the table below:

$\begin{array}{lccc} & \boldsymbol{b}(\mathrm{kb} / \mathrm{s}) & \boldsymbol{\rho} & C(\mathrm{Mb} / \mathrm{s}) \\ \text { Exp1 } & (64,64,64) & (\gamma, 2 \gamma, 3 \gamma) & 2 \gamma \\ \text { Exp2 } & (64,128,512,1000) & (8 \gamma, 4 \gamma, \gamma, \gamma) & 2 \gamma\end{array}$

The parameter $\gamma$ represents the scaling factor (multiplier), which is used to evaluate PDAC behavior under large scale systems. Note that the limiting regime is reached when $\gamma \rightarrow \infty$. Each $\gamma$ gives a different problem whose solution (the partitions $C_{k}$ ) is obtained from (14). After obtaining the partitions we computed the blocking probabilities and assessed the adequacy of the solutions in terms of the errors produced (19). Fig. 1 shows PDAC errors for Exp1 where each curve represents the results obtained for a different weight setting. It can be observed that errors decrease as system scales, when the capacity is close to $2 \mathrm{Gbps}$ all errors fall below $1 \%$. A similar behavior was also observed in Fig. 2 for Exp2, however the system had to be scaled further to have all errors below $1 \%$. Both figures showed accurate implementations of PDAC with class partitions obtained from (14) as the scaling factor grows. Note that the main purpose of the scaling parameter was to show how precision is improved when load, i.e., number of flows (up to 32768 in Exp1 and below 327680 in Exp2) and capacity (BW) are scaled together.

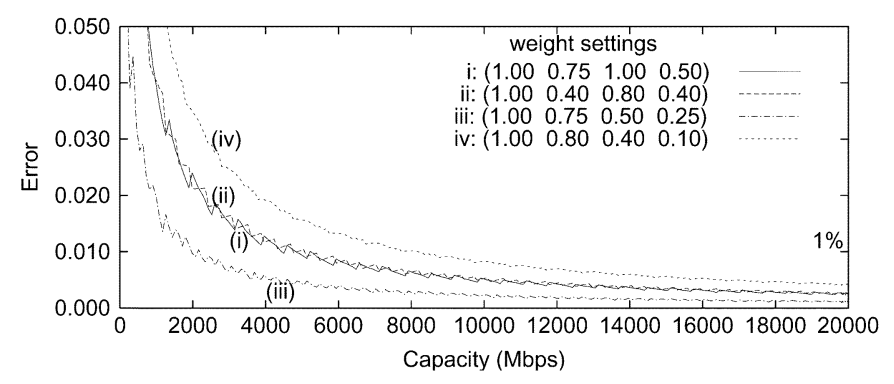

Fig. 2. PDAC errors for Exp2, system scaled from $\gamma=1$ to $\gamma=10^{4}$.

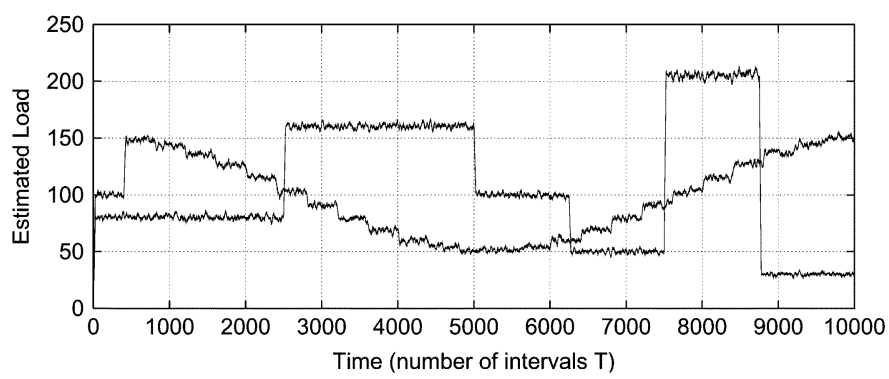

Fig. 3. Evaluation of the MLE in two different scenarios: loads changing abruptaly and with a cosine function.

We also evaluate the performance of the load estimator presented in Section III. Fig. 3 shows the results obtained for two different scenarios: loads changing abruptly and loads changing according to a cosine function. A new estimation is returned at each interval $T$, which was chosen to be five times the mean session holding time (exponentially distributed). A maximum error of $10.4 \%$ and mean error of $1.79 \%$ were observed. Other settings were also tested, including deterministic holding times, showing high accuracy and robustness in relation to the independent sample assumption.

\section{CONCLUSION}

We proposed a service, the PDAC, which controls the admittance of traffic from different classes according to a predefined set of weights. Such service conforms with the general idea behind the proportional differentiated model. PDAC is a scalable service since it uses a class-based approach where the necessary per-flow management procedures in a practical implementation are light. Given that PDAC involves the use of complex blocking probability equations we applied asymptotic approximation to simplify the framework. Also we relaxed PDAC definition to overcome feasibility problems imposed by the equality constraints in general PDS goal. We proposed an offered load estimator and briefly discussed a practical implementation of PDAC in the network. Finally, numerical results showed that our approximated solution was adequate, specially for large scale problems when the limiting regime approximation is precise.

\section{REFERENCES}

[1] C. Dovrolis, D. Stiliadis, and P. Ramanathan, "Proportional differentiated services: Delay differentiation and packet scheduling," IEEE/ACM Trans. Networking, vol. 10, pp. 12-26, Feb. 2002.

[2] K. W. Ross, Multiservice Loss Models for Broadband Telecommunication Networks. New York: Springer-Verlag, 1995.

[3] D. Jagerman, "Some properties of the Erlang loss function," Bell Syst. Tech. J., vol. 53, no. 3, pp. 525-551, 1974. 